\title{
Sesgo diagnóstico en época de COVID-19
}

\section{Diagnostic bias in COVID-19 times}

\begin{abstract}
Roberto Rodríguez-Rivas ${ }^{1}$, Guillermo Delgado-García², Arturo Abundes-Corona ${ }^{3,4}$ y Teresa Corona ${ }^{4,5 *}$
${ }^{1}$ Departamento de Neurología, Instituto Nacional de Neurología y Neurocirugía, Ciudad de México, Méx.; ${ }^{2}$ Unidad de Neuromodulación y Plasticidad Cerebral, Centro de Investigación y Desarrollo en Ciencias de la Salud, Universidad Autónoma de Nuevo León, Monterrey, N.L.; ${ }^{3}$ División de Neurología, American British Cowdray Medical Center, Ciudad de México, Méx.; ${ }^{4}$ Laboratorio Clínico de Enfermedades Neurodegenerativas, Instituto Nacional de Neurología y Neurocirugía, Ciudad de México, Méx.; ${ }^{5}$ División de Estudios de Posgrado, Facultad de Medicina, Universidad Nacional Autónoma de México, Ciudad de México, Méx. México
\end{abstract}

\section{Antecedentes}

Previo al advenimiento de la pandemia, se hicieron observaciones acerca de nuestra baja capacidad para detectar y/o predecir la emergencia de nuevos virus patógenos que pudieran afectar al ser humano, por lo que la identificación de áreas de riesgo para este tipo de brotes fue propuesta en 2018. Sin embargo, los sistemas de vigilancia necesarios para esta tarea no fueron incorporados en muchos países. En México se incorporaron estrategias extrapoladas de la experiencia previa obtenida de la pandemia de influenza H1N1. El distanciamiento social y la comunicación de riesgos fueron puestos en marcha nuevamente en marzo del 2020, mostrando picos de aumento de la enfermedad hasta la fecha, así como, la creación (en la capital) de un centro temporal específico para la enfermedad por coronavirus 2019 (COVID-19, coronavirus disease 2019)

La pandemia por la COVID-19 modificó de forma evidente las actividades y conductas cotidianas en el mundo entero. El sector médico no fue una excepción, ya que la mayor parte de las actividades del sector público giró en torno a la COVID-19. En este contexto, es posible que los médicos sufrieran de sesgo cognitivo al momento de hacer sus diagnósticos en la práctica médica y las situaciones de emergencia como la pandemia pudieron haberlos aumentado.
Desde que la Organización Mundial de la Salud (OMS) declaró que la COVID-19 se estaba comportando como una pandemia, el 11 de marzo del 2020, el mundo entero modificó sus actividades y conductas cotidianas, incluso algunos países cerraron sus fronteras. Los sistemas de salud y seguridad social se vieron imposibilitados para brindar la atención médica necesaria, especialmente en los países de ingresos bajos y medianos. Nadie estaba completamente preparado para recibir esta pandemia, la cual saturó los servicios de salud por la morbilidad y mortalidad asociadas a ella. Aún hoy día estamos experimentando sus consecuencias. Estas se han convertido también en un problema de atención médica, requiriendo en ocasiones de rehabilitación respiratoria, neurológica, psiquiátrica y otras más. Países como China construyeron centros de atención especializados parar mitigar los daños, sin embargo estas medidas no tuvieron el mismo impacto sobre la mortalidad en algunos países de América Latina. Países como Brasil y México registraron más de 220,000 muertes hasta la fecha.

Una vez iniciada la vacunación en noviembre del 2020, el planeta entero se llenó de esperanza; con avances más rápidos en países de mayor ingreso, la OMS hizo un llamado para compartir estos recursos con países más limitados económicamente, lo cual ha sucedió de manera irregular.

Los médicos en México atendemos diariamente pacientes que tienen o han padecido COVID-19, 
habitualmente realizamos un examen exhaustivo a cada uno de ellos, teniendo en mente las secuelas de esta infección en nuestros pacientes. Es en este punto cuando se presenta la posibilidad de que mantengamos un sesgo cognitivo al momento del diagnóstico. Los sesgos cognitivos han sido relacionados hasta con el $74 \%$ de los errores diagnósticos en la práctica médica y las situaciones de emergencia como la pandemia pueden haberlos aumentado, como queda patente en múltiples reportes de caso de esta situación en la literatura médica ${ }^{2,3}$.

La carga de enfermedades crónico-degenerativas y metabólicas parece haber aumentado durante la pandemia, tanto en incidencia como en complicaciones, y la reducción en la atención a estas situaciones, así como a otras enfermedades prevalentes en nuestro país, ha desviado la atención y el interés tanto de médicos como de autoridades, por lo que merecen un análisis riguroso $0^{4,5}$.

Con esto en mente, el abordaje diagnóstico debe conducirse con el mismo rigor clínico y científico que se hacía previo a la pandemia, con la finalidad de llegar al diagnóstico certero de las manifestaciones clínicas que presenta cada paciente, aun cuando hayan sido o sean positivos en las pruebas del coronavirus 2 del síndrome respiratorio agudo grave (SARS-CoV-2). Es importante reconocer a manera de introspección el riesgo de sesgo cognitivo en esta situación pandémica, ya que (de acuerdo con lo propuesto por Wilson y Breeke) el segundo paso en el proceso de eliminar estos sesgos es el identificarlos dentro de nuestro quehacer médico diario, seguido por encontrar la motivación adecuada para eliminarlos ${ }^{6}$.

\section{Conclusiones}

El diagnóstico situacional de salud de nuestro país muestra que, pese a que la COVID-19 ha cobrado innumerables vidas y se ha posicionado como primera causa de mortalidad materna, otros factores de riesgo y enfermedades crónico-degenerativas continúan siendo relevantes. El sesgo cognitivo puede retrasar un diagnóstico oportuno y el médico debe de estar en constante reflexión para evitarlo. Es importante hacer conciencia en el clínico del sesgo cognitivo que podemos padecer al abordar pacientes durante esta pandemia, y de la importancia de tener claro cuál es el panorama actual de las enfermedades que afectan a nuestra población.

\section{Financiamiento}

No se obtuvo ningún financiamiento externo para la realización de este manuscrito.

\section{Conflicto de intereses}

Los autores declaran no tener conflicto de intereses en el desarrollo o publicación de este manuscrito.

\section{Responsabilidades éticas}

Protección de personas y animales. Los autores declaran que para esta investigación no se han realizado experimentos en seres humanos ni en animales

Confidencialidad de los datos. Los autores declaran que han seguido los protocolos de su centro de trabajo sobre la publicación de datos de

Derecho a la privacidad y consentimiento informado. Los autores declaran que en este artículo no aparecen datos de pacientes.

\section{Bibliografía}

1. Vázquez RRV, Gallardo-Rincón H, Lomelín-Gascón J, Ville Benavides R, Juárez LM, Bello HH, et al. Impact of preemptive hospitalization on health outcomes at the temporary COVID-19 hospital in Mexico City: a prospective observational study. Ther Adv Infect Dis. 2021;8:20499361211040325.

2. O'sullivan ED, Schofield SJ. Cognitive bias in clinical medicine. J R Coll Physicians Edinb. 2018;48(3):225-32

3. DiMaria CN, Lee B, Fischer R, Eiger G. Cognitive bias in the COVID-19 pandemic. Cureus. 2020;12(7):e9019.

4. Rosenbaum L. The untold toll - The pandemic's effects on patients without Covid-19. N Engl J Med. 2020;382(24):2368-71.

5. Bornstein SR, Rubino F, Ludwig B, Rietzsch H, Schwarz PE, Rodionov RN, et al. Consequences of the COVID-19 pandemic for patients with metabolic diseases. Nat Metab. 2021;3(3):289-92.

6. Wilson TD, Brekke N. Mental contamination and mental correction: unwanted influences on judgments and evaluations. Psychol Bull. 1994;116(1):117-42. 